\title{
FATORES DE RISCO PARA PREMATURIDADE: PESQUISA DOCUMENTAL
}

\author{
Risk factors for prematurity: document search \\ Factores de riesgo para prematuridade: documento \\ de búsqueda
}

Helena Ângela de Camargo Ramos ${ }^{1}$
Roberto Kenji Nakamura Cuman ${ }^{2}$

\section{RESUMO}

Objetivou-se identificar o perfil de mães e de prematuros nascidos vivos e caracterizar os recém-nascidos prematuros em situação de risco para o crescimento e desenvolvimento. Estudo epidemiológico de corte transversal realizado em Guarapuava, PR. Os dados foram obtidos a partir do sistema de informações sobre nascidos vivos (SINASC) com base nas declarações de nascidos vivos. A análise estatística foi realizada em uma amostra composta por 106 declarações de nascidos vivos prematuros, de janeiro a junho de 2005, e suas respectivas mães. As variáveis utilizadas foram: características sociodemográficas, condições da gestação e parto e características dos prematuros nascidos vivos. Concluiu-se que conhecer e avaliar o perfil das mães e 0 número e a situação dos nascimentos de crianças de uma área, em um período de tempo, é importante na determinação dos riscos vitais relacionados a condições do nascimento, crescimento e desenvolvimento infantil, sendo esses aspectos componentes de vários indicadores de saúde e fundamentais para a assistência na área materno-infantil.

Palavras-chave: Prematuro. Mortalidade Infantil. Gravidez. Fatores de Risco.

\begin{abstract}
The objective was to identify the profile of mothers and premature births and characterize the premature newborns at risk for growth and development. A cross-sectional epidemiological study conducted in Guarapuava - PR. The data were obtained through the information system on live births (SINASC) based on declarations of live births. The statistical analysis was performed on a sample composed by 106 live births premature declarations, of January to June 2005 , and their mothers. The used variables were: characteristics sociodemographics, the gestation period and child-birth conditions, and characteristics of the alive born infants. It was concluded that knowing and evaluating the mothers' profile, the number and situation of the children's of a birth area, in a period of time, is important in the determination of the vital risks related to conditions of the birth, growth and infantile development, being these component aspects of several health indicators, and fundamental for the attendance in the maternal-infantile area.
\end{abstract}

Keywords:Premature. Infant mortality. Pregnancy. Risk factors.

\section{Resumen}

El objetivo fue identificar el perfil de las madres y los nacimientos prematuros y caracterizar los recién nacidos prematuros en riesgo para el crecimiento y el desarrollo. Un estudio epidemiológico transversal realizado en Guarapuava - PR. Los datos se obtuvieron a través del sistema de información sobre nacidos vivos (SINASC), basado en las declaraciones de los nacidos vivos. El análisis estadístico se realizó sobre una muestra compuesta por 106 nacidos vivos prematuro, declaraciones de enero a junio de 2005 , y sus madres. Las variables utilizadas fueron: características sociodemográficas, condiciones del embarazo y parto y características de los prematuros nacidos vivos. Se concluyo que conocer y estudiar el perfil de las madres, el número y condiciones del nacimiento de niños de una área, en periodo de tiempo son importantes en la determinación de los riesgos vitales relacionados a condiciones del nacimiento, crecimiento y desenvolvimiento infantil, siendo estos aspectos componentes de varios indicadores de salud y fundamentales para la asistencia materno-infantil.

Palabras clave: Prematuro. Mortalidad infantil. Embarazo. Factores de riesgo.

'Enfermeira, Mestra em Ciências da Saúde pela Universidade Estadual de Maringá - UEM. Professora do Departamento de Enfermagem. Centro de Ciências da Saúde. Universidade Estadual do Centro Oeste - UNICENTRO. Guarapuava- PR. Brasil. E-mail: helenaramosbr@hotmail.com, 2Farmacêutico, Doutor em Farmacologia pela Universidade de São Paulo - USP. Professor do Departamento de Farmácia e Farmacologia. Centro de Ciências Biológicas. Universidade Estadual de Maringá - UEM. Maringá - PR. Brasil. E-mail: rkncuman@uem.br 


\section{INTRODUCÃO}

A prematuridade é decorrente de circunstâncias diversas e imprevisíveis, em todos os lugares e classes sociais. Acarreta às famílias e à sociedade em geral um custo social e financeiro de difícil mensuração. Exige da estrutura assistencial capacidade técnica e equipamentos nem sempre disponíveis. Afeta diretamente a estrutura familiar alterando as expectativas e anseios que permeiam a perinatalidade. É difícil avaliar os componentes que influenciam e são influenciados pelo complexo processo do nascimento prematuro.

0 acompanhamento estatístico dos nascimentos foi um avanço significativo na área da epidemiologia: possibilitou conhecer e saber quem nasce, como nasce, onde nasce e em que condições nasce. Isso foi possível a partir de 1990, quando o Sistema de Informações de Nascidos Vivos (SINASC) foi implantado pelo Ministério da Saúde, por meio da Declaração de Nascido Vivo (DN), padronizada nacionalmente e preenchida nos hospitais e em outras instituições de saúde nos quais ocorrem partos, e nos Cartórios de Registro Civil para os partos domiciliares.

OSINASC propicia um aporte significativo de dados sobre a gravidez, o parto e as condições da criança ao nascer, com suas características mais importantes. Esses dados são relevantes para análises epidemiológicas, estatísticas, demográficas, e para a definição de prioridades das políticas de saúde. As informaç̃es são consolidadas pelos municípios e estados e compõem o "fechamento" da base nacional".

0 resultado esperado de uma gestação é a obtenção de recém-nascido (RN) sadio com mínimo trauma para a mãe. Em algumas situações, isso não é possível, devido a complicações durante a gravidez ou parto, ou com o concepto ${ }^{2}$. Essas intercorrências no processo do ciclo gravídico puerperal geram riscos à integridade da saúde tanto da mãe quanto do concepto e podem evoluir para a morte.

Tão importante como saber quantos nascem é saber quantos morrem, especialmente no primeiro ano de vida. Por isso, o coeficiente de mortalidade infantil é tão expressivo, pois evidencia uma série de fatores: conhecimento das mães, nível de imunização, disponibilidade de serviços de saúde, disponibilidade de renda e alimentos na família, disponibilidade de água limpa e saneamento básico, entre outros. Esse coeficiente é considerado a síntese da qualidade de vida e do nível de desenvolvimento de uma população. ${ }^{3-4}$.

A prematuridade como causa de mortalidade infantil tem sido estudada em diferentes países, e os estudos constatam que inúmeras são as causas que levam um bebê a nascer prematuro, especialmente as relacionadas ao aparelho genital feminino, alterações placentárias (placenta prévia e descolamento prematuro) e excesso de líquido amniótico. Outros fatores incluem: a idade materna (maior incidência em mães mais jovens), infecções maternas, primiparidade (mais frequente no primeiro filho). Porém, na maioria dos casos, a causa é desconhecida.
0 parto pré-termo é definido como aquele cuja gestação termina entre a $20^{\mathrm{a}}$ e a $37^{\mathrm{a}}$ semanas ou entre 140 e 257 dias após o primeiro dia da última menstruação $0^{5}$. A mortalidade e a morbidade neonatal são maiores entre os neonatos prematuros, além disso, a carga econômica associada a esses nascimentos é significativa na medida em que o parto prematuro demanda assistência e cuidados de maior nível de complexidade, especialmente com relação ao neonato ${ }^{6}$.

A imaturidade geral pode levar à disfunção em qualquer órgão ou sistema corporal, e o neonato prematuro também pode sofrer comprometimento ou intercorrências ao longo do seu desenvolvimento.

0 conhecimento das características de um grupo populacional contribui para a redução dos índices dos indicadores de saúde, principalmente o coeficiente de mortalidade infantil. Esses dados alicerçam, direcionam e subsidiam as ações propostas pelos diversos serviços de assistência inclusive a assistência à saúde, bem como sua forma de execução. Portanto, uma avaliação contínua desses indicadores de saúde pelas administrações públicas forneceria estratégias para assistência integral à criança e instrumentos para redução da incidência de morbimortalidade infantil.

Guarapuava é um município de médio porte situado na região centro-oeste do Paraná, cuja economia se baseia na agropecuária extensiva. Nos últimos anos, houve redução das altas taxas de mortalidade infantil, um dos seus principais problemas de saúde. Segundo fonte da Divisão de Epidemiologia da SMS, o índice de mortalidade infantil no ano de 2005 foi de 22,35. 0 índice preconizado pela OMS como aceitável é de 15 mortes para cada mil nascidos vivos.

A principal causa direta de mortes infantis no município no ano de 2005 foi a prematuridade; e as causas indiretas foram outros agravos e patologias. (SMS, Divisão de Vigilância Epidemiológica). Embora obscuras as suas causas, elas porém estão sempre vinculadas com os fatores de risco, o que nos faz refletir que estão diretamente relacionadas com o perfil e condições maternas, bem como com a assistência e cuidados prestados na gestação e parto.

A principal forma de intervir e prevenir agravos ou riscos é justamente o conhecimento e o monitoramento desses fatores, bem como das condições de nascimento, considerando o estado geral, as condições de saúde da mãe e a assistência prestada no processo do nascimento, principal marco do ciclo gravídicopuerperal.

A partir da década de 90, a maior visibilidade dos óbitos em recém-nascidos e os alarmantes índices de mortalidade materna foram responsáveis pela inclusão das estratégias de organização da atenção à gestante e ao recém-nascido na 
agenda de prioridades das políticas de saúde; entretanto, a análise da atenção ao prematuro em nosso meio revela necessidade de uniformidade na cober tura e confiabilidade na assistência. 0 acesso aos serviços de maior complexidade é difícil, em função da sua oferta insuficiente, o que também é verdadeiro no que se refere à quantidade e à qualidade de recursos humanos ${ }^{7}$.

A prematuridade tem se evidenciado no município como um dos principais problemas de saúde. É um importante fator que contribui para a elevada taxa de mortalidade infantil, acarretando danos e sequelas de difícil mensuração aos recémnascidos que conseguem superar o período neonatal de risco.

Essa sobrevida ocorre devido aos cuidados intensivos e tecnologias utilizadas, porém a manutenção dessa assistência tem um alto custo financeiro e depende da disponibilidade de recursos humanos preparados e capacitados para tal.

Consideramos que conhecer e compreender o complexo processo do nascimento e os fatores que nele interferem é fundamental para a assistência de qualidade efetiva ao binômio mãe-filho, bem como para otimizar e racionalizar o atendimento prestado em todas as etapas do ciclo reprodutivo, priorizando as ações de prevenção, recuperação e manutenção da vida. $E$ identificar fatores de risco associados ao ciclo gravídicopuerperal possibilita direcionar e adotar medidas preventivas e curativas de forma adequada à realidade.

0 objetivo deste trabalho foi identificar o perfil de mães e de prematuros nascidos vivos no município de Guarapuava, Paraná, e caracterizar os recém-nascidos prematuros em situação de risco para o crescimento e desenvolvimento.

\section{MATERIAIS E MÉTODOS}

Estudo epidemiológico de corte transversal, realizado no município de Guarapuava, PR. A amostra foi composta da totalidade dos prematuros vivos (106) e suas respectivas mães (101), no período de janeiro a junho do ano de 2005. Foram excluídas as mães de nascidos mortos e as mães de bebês nascidos vivos residentes em outro município. As mães e seus conceptos foram identificados e caracterizados de acordo com os dados da DN/MS.

A coleta de dados foi realizada por meio da busca de documentos com base no formulário DN/MS (Declaração de nascidos vivos) nos arquivos do Departamento de Vigilância Epidemiológica da Secretaria Municipal de Saúde do município, e foram utilizadas informações das bases de dados vitais do Ministério da Saúde do Sistema de Informações sobre Nascidos Vivos (SINASC). Os dados obtidos foram analisados pelo software estatístico Statistic 7.0.

As variáveis do perfil materno utilizadas foram: idade, escolaridade, ocupação principal, estado civil, local de residência, duração da gestação, tipo de gravidez, tipo de parto e número de consultas de pré-natal.

As variáveis de caracterização dos recém-nascidos foram: sexo, raça, índice de Apgar no quinto minuto de vida, peso ao nascer e má formação congênita .

Quanto aos aspectos éticos da pesquisa, foram observados os disciplinados pela Resolução 196/96 do Conselho Nacional de Saúde - Ministério da Saúde. Foi solicitada autorização para a realização do estudo à Secretaria de Saúde do Município de Guarapuava, PR. 0 projeto foi aprovado pelo Comitê de Ética da Universidade Estadual do Centro-Oeste - UNICENTRO, protocolado sob o n 95/2006.

\section{RESULTADOS E DISCUSSÃO}

As variáveis foram agrupadas em três blocos, compondo três tabelas de dados: a primeira, refere-se ao perfil materno; a segunda, refere-se às condições da gestação e parto; e a terceira compõe as características dos prematuros.

Tabela 1 - Características demográficas das Mães de prematuros nascidos vivos no município de Guarapuava, PR, 2005.

\begin{tabular}{lcc}
\hline Variáveis & Frequência & \\
\hline & & \\
Idade (anos) & & 4,00 \\
$>15$ anos & 04 & 30,00 \\
$15-19$ & 30 & 54,00 \\
$20-34$ & 55 & 12,00 \\
$35-45$ & 12 & \\
& & 51,00 \\
Estado Civil & & 37,00 \\
Solteira & 52 & 1,00 \\
Casada & 37 & 1,00 \\
Viúva & 01 & 10,00 \\
União Consensual & 01 & \\
Ignorado & 10 & 17,00 \\
Escolaridade & & 30,00 \\
De 1 a 3 anos & & 38,00 \\
De 4 a 7 anos & 17 & 13,00 \\
De 8 a 11 anos & 30 & 2,00 \\
12 a mais & 39 & \\
Ignorado & 13 & \\
Ocupação & 02 & 71,00 \\
Do Lar & & 17,00 \\
Assalariada & & 6,00 \\
Profissional autônoma & 72 & 3,00 \\
Estudante & 17 & 100,00 \\
Agricultora & 06 & \\
Total & 03 & \\
\hline
\end{tabular}


Prematuridade e fatores de risco

Tabela 2 - Condições da gestação e parto de prematuros nascidos vivos no município de Guarapuava, PR, 2005.

\begin{tabular}{|c|c|c|}
\hline Variáveis & Frequência & $\%$ \\
\hline $\begin{array}{l}\text { Tempo da Gestação } \\
\text { De } 22 \text { a } 27 \text { semanas } \\
\text { De } 28 \text { a } 31 \text { semanas } \\
\text { De } 32 \text { a } 36 \text { semanas }\end{array}$ & $\begin{array}{l}06 \\
20 \\
75\end{array}$ & $\begin{array}{l}6,00 \\
20,00 \\
74,00\end{array}$ \\
\hline $\begin{array}{l}\text { Tipo de Gravidez } \\
\text { Única } \\
\text { Dupla }\end{array}$ & $\begin{array}{c}96 \\
5\end{array}$ & $\begin{array}{r}95,00 \\
5,00\end{array}$ \\
\hline $\begin{array}{l}\text { Tipo de Parto } \\
\text { Vaginal } \\
\text { Cesáreo }\end{array}$ & $\begin{array}{l}59 \\
42\end{array}$ & $\begin{array}{l}58,00 \\
42,00\end{array}$ \\
\hline $\begin{array}{l}\text { Consultas Pré-Natal } \\
\text { Nenhuma } \\
\text { De } 1 \text { a } 3 \\
\text { De } 4 \text { a } 6 \\
7 \text { e mais }\end{array}$ & $\begin{array}{l}03 \\
14 \\
52 \\
32\end{array}$ & $\begin{array}{l}3,00 \\
14,00 \\
51,00 \\
32,00\end{array}$ \\
\hline $\begin{array}{l}\text { Localização } \\
\text { Área urbana (Bairo) } \\
\text { Área urbana (Periferia) } \\
\text { Zona Rural (Nilarejo) } \\
\text { Zona Rural (Interior) }\end{array}$ & $\begin{array}{l}49 \\
32 \\
12 \\
08\end{array}$ & $\begin{array}{l}48,00 \\
32,00 \\
12,00 \\
8,00\end{array}$ \\
\hline Total & 101 & 100,00 \\
\hline
\end{tabular}

Tabela 3 - Características dos Prematuros nascidos vivos no município de Guarapuava, PR, 2005.

\begin{tabular}{|c|c|c|}
\hline Variáveis & Frequência & $\%$ \\
\hline \multicolumn{3}{|l|}{ Sexo } \\
\hline Masculino & 51 & 48,00 \\
\hline Feminino & 55 & 52,00 \\
\hline \multicolumn{3}{|l|}{ Raça } \\
\hline Branca & 100 & 94,00 \\
\hline Preta & 01 & 1,00 \\
\hline Amarela & 01 & 1,00 \\
\hline Parda & 02 & 2,00 \\
\hline Ignorada & 02 & 2,00 \\
\hline \multicolumn{3}{|l|}{ Apgar } \\
\hline$<5$ & 04 & 4,00 \\
\hline 5 a 6 & 14 & 13,00 \\
\hline 7 a 8 & 28 & 27,00 \\
\hline 9 a 10 & 56 & 52,00 \\
\hline Ignorado & 04 & 4,00 \\
\hline \multicolumn{3}{|l|}{ Peso ao nascer } \\
\hline$<1.000 \mathrm{~g}$ & 10 & 9,00 \\
\hline 1.000 a $1.999 \mathrm{~g}$ & 44 & 42,00 \\
\hline 2.000 a $2.499 \mathrm{~g}$ & 29 & 27,00 \\
\hline 2.500 a $3.000 \mathrm{~g}$ & 17 & 16,00 \\
\hline 3.100 a $3.499 \mathrm{~g}$ & 04 & 4,00 \\
\hline$>3.500 \mathrm{~g}$ & 01 & 1,00 \\
\hline Ignorado & 01 & 1,00 \\
\hline \multicolumn{3}{|l|}{ Malformação Congênita } \\
\hline Sim & 02 & 2,00 \\
\hline Não & 103 & 97,00 \\
\hline Ignorado & 01 & 1,00 \\
\hline Total & 106 & 100,00 \\
\hline
\end{tabular}


Os resultados quanto ao perfil materno (Tabela 1) foram: com relação à idade, $54 \%$ estavam na faixa etária entre $20 \mathrm{e}$ 34 anos, considerado período reprodutivo; $12 \%$ estavam na faixa etária entre 35 e 45 anos; 30\%, na faixa etária entre 15 e 19 anos; e 4\%, na faixa etária igual ou menor que 15 anos. Somando-se as duas últimas faixas etárias, ou seja, todas as menores de 19 anos, consideradas adolescentes, constatou-se $34 \%$ da amostra.

A gravidez na adolescência é fator de maior concentração de agravos à saúde materna, bem como de complicações perinatais, tais como baixo ganho de peso materno, desproporção cefalopélvica, pré-eclampsia, prematuridade, baixo peso ao nascer e Apgar baixo no quinto minuto ${ }^{8}$.

As intercorrências relativas à gravidez na adolescência se potencializam quando associadas a condições socioeconômicas e geográficas, bem como à fragilidade da estrutura familiar e dificuldade de acesso aos serviços assistenciais.

Com relação ao estado civil, encontramos: $10 \%$ de situação conjugal ignorada; $1 \%$ de união consensual; $1 \%$ de viuvez; $37 \%$ eram casadas; e $51 \%$, solteiras. Esse resultado indica que a maioria das mães não tem apoio de um companheiro e, portanto, não tem com quem dividir ou compartilhar suas dificuldades e responsabilidades. Essa realidade evidencia as mudanças estruturais que a família vem passando ao longo dos anos, motivada pelas condições sociais, econômicas e culturais, não apresentando na atualidade, em sua maioria, uma composição paternalista. Independentemente de sua estrutura e composição, deve-se considerar a importância do envolvimento da família na promoção à saúde e cuidado a seus membros.

Em estudo correlacionando a relação marital da mãe, a mortalidade infantil e aspectos socioeconômicos demonstraram que a taxa de mortalidade infantil é maior para crianças de mães solteiras, com exceção das de etnias de raça branca, que têm alto grau de instrução. Além disso, entre as mães solteiras e as casadas, independentemente da etnia, a taxa de mortalidade infantil é mais alta entre os filhos de mães solteiras 9 .

0 acesso a informações e a medidas de prevenção e promoção à saúde é fundamental, de forma que os baixos índices de escolaridade impedem as pessoas de adequarem sua vida pessoal e reprodutiva. A falta de políticas e de mecanismos que assegurem a todos os segmentos 0 acesso à escola, associada à evasão escolar e à repetência, está sempre associada a precárias condições socioeconômicas. Nesse sentido, a educação e a formação informal, pela qual se pode realizar promoção de conhecimentos e práticas de saúde, aliadas à escola e a outras instituições, promovem a busca de transformações sociais ${ }^{10}$.

Em nosso estudo, quanto à escolaridade, os resultados indicaram que: em $2 \%$, o grau de escolaridade é ignorado; $13 \%$ têm mais de 12 anos de estudo; $38 \%$ têm entre 8 e 11 anos de estudo; $30 \%$, entre 4 e 7 anos de estudo; e $17 \%$, entre 1 e 3 anos de estudo. Quanto à escolaridade considerada baixa (ensino fundamental incompleto, tempo de estudo igual ou menor que 7 anos), observamos que $47 \%$ não têm sequer estudo fundamental completo.

Podemos considerar que, em geral, a baixa escolaridade está associada ao baixo padrão socioeconômico, fator que pode predispor a situações potencialmente de risco para a mãe e 0 recém-nascido, além de impedir o acesso a informações e orientações, restringir a capacidade de cuidado e assistência, dificultar o exercício de direitos e de cidadania. A situação constatada comprova que o nível de escolaridade interfere diretamente nas condições de vida e saúde das pessoas e confirma a correlação existente: quanto menor a escolaridade, maior a dificuldade de entendimento da necessidade de cuidados especiais durante a gestação, levando ao início tardio e ausência ao pré-natal, alimentação inadequada e hábitos e vícios incompatíveis com a gravidez.

No que se refere à ocupação, o estudo revelou que $3 \%$ da amostra eram de agricultoras; $3 \%$, estudantes; $6 \%$, profissionais autônomas; $17 \%$, assalariadas; e $71 \%$ apresentaram ocupação do lar, ou seja, trabalho doméstico sem remuneração.

0 ser humano está exposto aos riscos inerentes às posições ocupadas no espaço social; saúde-doença é também consequência do acesso a esses capitais, uma vez que muitas diferenças, que geralmente se associam ao efeito do espaço geográfico, por exemplo, à oposição centro-periferia, são efeitos da distância no espaço social, isto é, da distribuição desigual das diferentes espécies de capital no espaço geográfico ${ }^{10}$.

Quanto à localização da moradia, $8 \%$ residem em zona rural (interior); $12 \%$ residem em zona rural (vilarejo); $32 \%$ residem em área urbana (periferia); e $48 \%$ residem em área urbana (bairros). Esses percentuais podem estar associados à questão do acesso aos serviços de saúde, uma vez que, se considerarmos estrutura de atendimento, localização e qualificação dos serviços, constatamos que, somados, os percentuais vão compor $52 \%$ que residem em zona rural e/ou periferia, ou seja, onde o acesso aos bens e serviços é restrito. Os fatores ambientais podem ser determinantes da taxa de mortalidade infantil. Uma pesquisa realizada observou que as crianças nascidas no meio urbano apresentaram menor coeficiente de mortalidade infantil em relação àquelas que nasceram no meio rural. A ausência de saneamento básico e a poluição excessiva do ar podem também ser responsáveis pelo aumento dessa taxa ${ }^{11}$.

A avaliação de risco gestacional deve ser realizada em todas as consultas, seguindo os critérios para sua caracterização, e a gestante deve ser encaminhada ao pré-natal adequado à sua situaçãa $0^{12}$. A ausência de cuidados pré-natais está associada a um aumento do risco de baixo peso ao nascer, partos prematuros e mortalidade materna e infantil ${ }^{11}$. No Paraná, 0 calendário ideal adotado para o acompanhamento do pré-natal sugere: uma consulta mensal até a $30^{\mathrm{a}}$ semana, consultas 
quinzenais da $31^{\mathrm{a}}$ a $36^{\mathrm{a}}$ semana, consultas semanais da $37^{\mathrm{a}}$ a $40^{\mathrm{a}}$ semana, consulta a cada três dias após a $40^{\mathrm{a}}$ semana, com limite máximo até a $42^{\mathrm{a}}$ semana. Para uma gestante sem fatores de riscos detectados na primeira consulta, estabelece-se que, no mínimo, seis consultas sejam realizadas pelo médico ou enfermeira ${ }^{12}$.

Com relação ao número de consultas de acompanhamento pré-natal, o estudo demonstrou que $32 \%$ realizaram sete ou mais consultas, $51 \%$ realizaram de 4 a 6 consultas, $14 \%$ realizaram de 1 a 3 consultas e $3 \%$ não realizaram nenhuma consulta. Nossos resultados indicam que, apesar da padronização no serviço de saúde para a realização do acompanhamento do pré-natal, ainda há baixa adesão a esse protocolo.

O Brasil ocupou, pouco tempo atrás, a nada invejável posição de campeão mundial de operações cesarianas. Se por um lado a cesárea realizada por razões médicas tem um grande potencial de reduzir a morbimortalidade materna e perinatal, por outro lado, o exagero de sua prática tem efeito oposto. Essa distorção é determinada por múltiplos fatores, históricos, estruturais, conjunturais ${ }^{13}$. Observamos que, quanto ao tipo de parto, $58 \%$ foram partos normais e $42 \%$ cesáreos. Os dados obtidos em nossa pesquisa indicam um percentual elevado de cesáreas, talvez porque os partos prematuros já incidem em risco por si só, potencializados pelos fatores idade, escolaridade, condições socioeconômicas maternas e gemelaridade, e, portanto têm indicação de intervenção cirúrgica. A desvalorização do parto normal e a prática de intervenções cirúrgicas desnecessárias, cada vez em maior número e frequência, é um indicativo da falta de informação e educação em saúde da população feminina, que se sente incapaz de escolher ou determinar práticas quando confrontadas com a opinião técnica dos profissionais de saúde ${ }^{14}$.

As taxas de prevalência do trabalho de parto pré-termo têm se mantido estável em torno de $10 \%$ de todos os partos. ${ }^{6}$ Estima-se que, anualmente, no mundo, 13 milhões de crianças nasçam prematuras, e o nascimento pré-termo continua sendo uma importante causa de mortalidade perinatal. Em nossa pesquisa, quanto às condições da gestação e parto (Tabela 2) e em relação ao tempo da gestação, observamos que, para $74 \%$ dos nascidos, esse período foi de 32 a 36 semanas; $20 \%$ tiveram tempo de gestação de 28 a 31 semanas; e para $6 \%$ o tempo de gestação foi de 22 a 27 semanas. Além disso, considerando-se prematuridade extrema, ou seja, tempo de gestação menor que 31 semanas, foi observada uma frequência de $26 \%$ para os prematuros nascidos vivos. De fato, cerca de $75 \%$ das mortes neonatais estão relacionadas ao parto prétermo ou às manifestações no recém-nascido pré-termo ${ }^{6}$.

A frequência de gêmeos tem crescido muito nos últimos anos, principalmente pelas técnicas de tratamento da infertilidade. A simples presença de mais um feto aumenta a chance de fetos prematuros, hipertensão arterial, ruptura das membranas e morte fetal intraútero ${ }^{15}$. Em nossa pesquisa, observamos que, quanto ao tipo de gravidez, $95 \%$ dos partos foram de gravidez única, enquanto $5 \%$ foram de gravidez dupla. A gemelaridade é um fator de risco para os nascimentos de baixo peso ao nascer, podendo ser considerada como um fator de risco para a mortalidade neonata ${ }^{11}$.

Várias teorias já foram sugeridas a fim de explicar os mecanismos determinantes da gemelaridade. Além da herança genética, fatores ambientais como idade materna, paridade, grupo étnico, antecedentes reprodutivos, uso de anticoncepcionais, tratamento para indução da ovulação, classe social e estatura materna foram descritos como predisponentes.

A incidência da gemelaridade é significativa proporcionalmente ao total dos nascimentos prematuros em questão, e potencializa por si só todos os riscos e situações já considerados anteriormente neste estudo. A gravidez múltipla aumenta os riscos de complicações no período gestacional especialmente para hipertensão arterial e para parto cesáreo.

Quanto às características dos prematuros (Tabela 3), relativas ao sexo, nasceram $52 \%$ do sexo feminino e $48 \%$ do sexo masculino. Apesar de este estudo mostrar predomínio de nascimentos de prematuros do sexo feminino, a questão de gênero não é significativa. Por outro lado, estudos realizados na Holanda demonstraram em seus resultados que o sexo masculino para o recém-nascido estava associado ao aumento do risco de sofrimento fetal, enquanto foi observado efeito protetor do sexo feminino?.

A diferença étnica pode estar ligada às condições socioeconômicas, que é um fator que interfere na mortalidade infantil. Em nossa pesquisa, observamos que, quanto à raça, $2 \%$ são de raça ignorada; $2 \%$ são de raça parda; $1 \%$, de raça amarela; $1 \%$, de raça preta; e $94 \%$, de raça branca. Neste estudo especificamente ocorre maior incidência de prematuridade na raça branca; isso não é relevante, pois é a raça da maioria da população residente. A predominância da raça branca se justifica pelo fato de que o município de Guarapuava foi colonizado por europeus (alemães, ucranianos e italianos), e a raça negra tem baixa incidência na região.

Vários estudos sobre a influência da etnia confirmam que, com relação aos fatores maternos, segundo a etnia, os filhos de mães negras apresentaram maiores taxas de mortalidade com relação a gemelaridade, peso ao nascer, prematuridade e situação socioeconômica. As taxas de mor talidade infantil de todas as etiologias foram maiores em mulheres negras e jovens ${ }^{9}$.

No que se refere ao índice de Apgar no quinto minuto de vida, o resultado da pesquisa mostra: $4 \%$ índice de Apgar ignorado; $52 \%$ índice de Apgar entre 9 e 10; 27\% índice de Apgar entre 7 e 8; $13 \%$ índice de apgar entre 5 e 6; e 4\% índice de Apgar igual ou menor que 5. Esse dado é interessante, pois revela que, entre todos os prematuros, um percentual de $17 \%$ obteve índice de Apgar insatisfatório no quinto minuto de vida. E somando-se as faixas com índice satisfatório, temos $79 \%$, indicando que, mesmo que tenham nascido com dificuldades, houve recuperação rápida na avaliação após 5 
minutos e isso indica um bom prognóstico e grandes chances de sobrevida. Os resultados obtidos demonstram também que $17 \%$ dos nascidos vivos tiveram a necessidade de atendimento de maior nível de complexidade e maiores chances de complicações e sequelas em vários níveis, remetendo à disponibilidade de estrutura técnica, equipamentos e recursos humanos capacitados para atendimento de maior complexidade e reversão da situação de risco apresentada ao nascer.

0 índice de Apgar baixo é útil para identificar os recémnascidos que necessitam de cuidados adicionais. Um índice de Apgar de 7 a 10 significa que o bebê é sadio e que provavelmente não terá problemas futuros. 0 índice de Apgar inferior a 7 é sinal de alerta de acordo com a alteração fisiopatológica e da maturidade do concepto ${ }^{2}$.

Com relação ao peso do nascimento, temos que: $1 \%$ teve peso ignorado; $1 \%$, peso maior que $3.500 \mathrm{~g} ; 4 \%$, peso entre 3.100 e $3.499 \mathrm{~g} ; 16 \%$, peso entre 2.600 e $3.000 \mathrm{~g} ; 27 \%$, peso entre 2.000 e $2.499 \mathrm{~g} ; 42 \%$. entre 1.000 e $1.999 \mathrm{~g} ; \mathrm{e}$ $9 \%$ menor que 1.000 g. 0 que demonstra que $22 \%$ dos nascidos vivos prematuros apresentaram peso normal ao nascimento $\mathrm{e}$ $78 \%$ apresentaram baixo peso ao nascer, sendo que, desses, $17 \%$ apresentaram peso insuficiente (PIN), $29 \%$ baixo peso ao nascer (BPN) e $54 \%$ muito baixo peso ao nascer (MBPN).

Quanto ao peso ao nascer, a organização Mundial da Saúde (OMS) define como BPN o recém-nascido com peso de nascimento menor que $2.500 \mathrm{~g}$, independente da idade gestacional. 0 BPN foi detectado como um fator determinante importante da desnutrição, reflete as condições nutricionais tanto do recém-nascido como da gestante, influencia o crescimento e desenvolvimento da criança e, em longo prazo, repercute nas condições de saúde do adulto ${ }^{16}$.

0 baixo peso ao nascer sempre foi motivo de preocupação para os profissionais da área de saúde, por se associar à maior morbimortalidade neonatal e infantil. 0 grupo de recémnascidos com peso menor que $2.500 \mathrm{~g}$ é heterogêneo, pois decorre de duas condições adversas, prematuridade ou restrição do crescimento intrauterino, que podem atuar de forma isolada ou sinérgica e em graus variáveis. 0 peso ao nascer representa o fator de risco que mais influencia a sobrevivência infantil ${ }^{16-17}$.

Quanto à má formação congênita, o estudo evidenciou a sua presença em $2 \%$ dos nascidos vivos, o que está de acordo com a incidência observada em pesquisas na literatura. A má formação é outro fator associado à mortalidade neonatal, e sua prevalência está entre $2 \%$ e $6 \%$, dependendo da população. Houve estudos em que essa taxa foi de $1,2 \%$, mostrando ainda que as más formações foram responsáveis por 9,2\% da mortalidade perinatal, por $12,8 \%$ da mortalidade neonatal e $45 \%$ das mortes infantis, sendo as principais más formações congênitas a doença congênita do coração e a hipoplasia pulmonar'.

A prematuridade também interfere na convivência familiar, no relacionamento, na proximidade, nos cuidados e na amamentação. As mães, ao se defrontarem com a vivência da hospitalização de seu filho, se deparam com as impossibilidades citadas e mostram-se ansiosas, com dúvidas e dificuldades diante dessa realidade ${ }^{17}$.

0 aumento da sobrevivência de prematuros cada vez menores e mais imaturos impõe o questionamento quanto à qualidade de sua vida futura. 0 prognóstico de desenvolvimento dos prematuros depende de complexa interação de fatores biológicos e ambientais atuantes no cérebro imaturo e vulnerável destas crianças $^{18}$.

\section{CONCLUSÕES}

A etiologia do parto prematuro é multifatorial. Há que se considerar a preocupação constante com os recém-nascidos prematuros bem como com as condições perinatais que sobre eles repercutem. A fragilidade dos recém-nascidos prematuros contribui para a possibilidade eminente de riscos, agravos e sequelas de diversos tipos com diferentes consequências e interveniências no processo do desenvolvimento e crescimento infantil. Portanto, faz-se necessário prever e considerar riscos e prognósticos para que se possa eventualmente instaurar e promover medidas preventivas.

0 estudo comprovou que o perfil de mães dos prematuros e a caracterização dos nascidos vivos são influenciados pelas condições sócias, econômicas e sanitárias da localidade em questão onde ocorrem a gestação e o nascimento, e que essas mesmas condições certamente influenciarão na qualidade de vida futura, sendo um fator contribuinte para os altos índices de mortalidade infantil, um dos principais problemas evidenciados no município.

Os dados mostram a importância de identificação dos principais fatores de risco de óbito para período neonatal, reafirmando a influência dos fatores biológicos fortemente relacionados ao componente neonatal da mortalidade infantil.

Nesse contexto, o acesso a informações, o conhecimento sobre a realidade, bem como o acompanhamento constante dos dados, taxas e índices pelos profissionais de saúde, certamente interferem nas ações desenvolvidas e contribuem no modo de cuidar e assistir à vida. Por outro lado, o acesso à informação em muitas situaç̃oses e localidades é limitado e difícil, por isso a Declaração de Nascido Vivo (DN) torna-se tão importante, pois é uma fonte riquíssima de dados que, dependendo da forma como são entendidos e interpretados e de sua aplicação no dia-a-dia, podem contribuir diretamente como banco de dados e ser subsídio para planejamento e alicerce de ações de assistência e de prevenção tanto na área da saúde pública quanto na área de assistência hospitalar. Atuar estrategicamente em uma localidade com o objetivo de intervir nos agravos à saúde implica planejamento das ações, e, portanto, faz-se necessário utilizar indicadores demográficos, epidemiológicos, político-sociais e econômicos a fim de realizar análise e diagnóstico situacional da realidade.

Vivemos na era da globalização, do avanço do conhecimento técnico e científico, da divulgação de recentes e fantásticas 
descobertas, porém permanece o maior desafio à humanidade: o controle da morbimortalidade, da manutenção da saúde, do uso de conhecimentos e de instrumentos e formas capazes de garantir a vida.

Deparamo-nos com situações enigmáticas, imprevisíveis e multifatoriais que interferem e contribuem para a antecipação dos nascimentos, corroborando a alta incidência de prematuridade. Como agravantes, constatamos a deficiência da estrutura existente para 0 atendimento e manutenção da saúde, os recursos técnicos e humanos inadequados e que não conseguem atender à demanda, o cuidado familiar deficiente e a falta de acompanhamento especializado adequado, predispondo esses recém-nascidos aos agravos e intercorrências inerentes ao crescimento e desenvolvimento humano.

Evidenciamos que as condições socioculturais, educacionais e econômicas determinam as condições do nascer, desenvolver, adoecer e morrer.

\section{REFERÊNCIAS}

1. Ministério da Saúde (BR). Para entender a gestão do SUS: CONASS. Brasília (DF); 2003.

2. Cunha AL, Fernandes DS, Melo PF, Guedes MH. Fatores associados à asfixia perinatal. Rev Bras Ginecol Obstet 2004 nov/dez; 26 (10): 799-805.

3. Miranda MIF, Ferriani MGC. Políticas públicas sociais para crianças e adolescentes. Goiânia(GO): AB; 2001.

4. Oliveira RB, Melo ECP, Knupp VMAO. Perfil dos óbitos infantis no município do Rio de Janeiro segundo peso ao nascer, no ano de 2002. Esc Anna Nery Rev Enferm 2008 mar; 12 (1): 25 -9.

5. Ramos JGL, Martins SHC, Valério EG, Muller ALL.Nascimento prétermo. In: Freitas F, Martins SHC, Ramos JGL, Magalhães JÁ. Rotinas em obstetrícia. Porto Alegre(RS): Artmed; 2001.p. 69 - 80.

6. Kenner C. Enfermagem neonatal. Rio de Janeiro (RJ): Reichmann \& Affonso; 2001.

7. Carvalho M, Gomes MASM. A mortalidade do prematuro extremo em nosso meio: realidade e desafios. J Pediatr 2005 mar/abr; 81(1): 111-18.

8. Goldenber GP, Figueiredo MCT, Silva RS. Gravidez na adolescência, pré-natal e resultados perinatais em Montes Claros, Minas Gerais, Brasil. Cad Saude Publica 2005 jul/ago; 21 (4): 1077 - 85.

9. Kozu KT, Godinho LT, Muniz MVF, Chiarioni P. Mortalidade infantil: causas e fatores de risco-um estudo bibliográfico. Homepage do Medstudents [on-line] 2006. Disponível em
A manutenção de indicadores de saúde aceitáveis implica concentração de esforços e recursos que, a médio e longo prazo, resultem em modificações das condições de vida e saúde das pessoas, traduzindo-se em qualidade de vida. Essa qualidade não está atrelada somente às questões relacionadas ao processo saúde/doença, mas reflete diretamente as condições do nascer, sobreviver e existir.

Os dados obtidos, em conjunto, permitiram concluir que conhecer e avaliar o perfil das mães e o número e a situação do nascimento de crianças de uma área, em um período de tempo, é importante na determinação dos riscos vitais relacionados a condições do nascimento, crescimento e desenvolvimento infantil, sendo esses aspectos componentes de vários indicadores de saúde e fundamentais para a assistência na área materno-infantil.

http://www.medstudents.com.br/original/original/mortinf/mortinf.htm

10. Ministério da Saúde (BR). Adolescer compreender atuar acolher. Projeto Acolher. Brasília (DF): ABEn; 2001.

11. Parker JD, Schoendorf KC, Kiely JL. A comparison of recent trends in infant mortality among twins and singletons. Pediatr Perin Epidemiol 2001 Jan; 1 (15):12-18.

12. Secretaria de Estado da Saúde (PR). Natural é o parto normal: pré-natal, parto e puerpério. $3^{\mathrm{a}}$ ed. Curitiba (PR); 2004.

13. Ministério da Saúde (BR). Secretaria de Políticas de Saúde. Área Técnica de Saúde da Mulher. Parto, aborto e puerpério: assistência humanizada à mulher. Brasília (DF); 2003.

14. Marque FC, Dias IMV, Azevedo L. A percepção da equipe de enfermagem sobre humanização do parto e nascimento. Esc Anna Nery Rev Enferm 2006 dez; 10(3): 439 - 47.

15. Rodrigues CT, Ferreira ID, Nordeste A, Fonseca M, Taborda A, Silva IS, Almeida MC. Epidemiologia da gestação múltipla casuística de 15 anos. Acta Med Port 2005; 18: 107 -11.

16. Motta ME, Silva GA, Araújo OC, Lira PI, Lima MC. 0 peso ao nascer influencia o estado nutricional ao final do primeiro ano de vida? J Pediatr 2005 set/out; 81(5):377- 82.

17. Gorgulho FR, Pacheco STA. Amamentação de prematuros em uma unidade neonatal: a vivência materna. Esc Anna Nery Rev Enferm 2008 mar; 12(1): 19 - 24

18. Rugolo LM. Crescimento e desenvolvimento a longo-prazo do prematuro extremo. J Pediatr 2005 mar/abr; 81(1)101-10. 